\title{
Differential diagnosis between statin myotoxicity and inflammatory myositis - case presentation
}

\author{
Andreea Daniela Tudorancea', Paulina Lucia Ciurea', Cristina Dorina Parvanescu', \\ Sineta Cristina Firulescu', Catalin Bogdan'2 Elena Madalina Vintila ${ }^{3}$, \\ Roxana Mihaela Dumitrascu', Cristina Ene ${ }^{1}$, Cristina Criveanu', \\ Florentin Ananu Vreju' ${ }^{1}$, Stefan-Cristian Dinescu ${ }^{1}$ \\ ${ }^{1}$ Department of Rheumatology, University of Medicine and Pharmacy Craiova, Romania \\ ${ }^{2}$ Department of Functional Sciences, University of Medicine and Pharmacy of Craiova, Romania \\ ${ }^{3}$ Department of Gastroenterology and Hepatology, Emergency County Hospital Craiova, Romania
}

\begin{abstract}
Inflammatory myopathies, include polymyositis, dermatomyositis, inclusion body myositis and necrotising myopathy, but their diagnosis requires a comprehensive differential, in order to optimise treatment and to have the best outcome. One of the most controversial diagnosis in this situation is drug related myotoxicity, since the symptoms may vary significantly, but usually include muscle weakness and myalgia accompanied by elevated creatine kinase serum levels

Patient background. We report a case of a 70 year-old patient, treated with statins, with onset of symptoms since one year with tolerable myalgia, accompanied by mild muscle weakness shortly after and progressive worsening in the last couple of months. Interruption of statins was recommended based on current symptoms and elevated muscle enzymes: creatine kinase (CK) x3 fold and aspartate aminotransferase (AST) x2 fold normal range. Investigations. Autoimmunity panel including anti-nuclear and 3-hydroxy-3-methylglutaryl-coenzyme A reductase (HMGCR) antibodies was negative. The needle EMG was abnormal, with diffuse fibrillation potentials in almost all investigated sites, both in the proximal and distal muscles. Complex repetitive discharges were also observed in most muscles tested. Existence of clear myogenic signs on needle EMG revealed the probable cause for the clinical presentation as being myogenic in nature.

Discussion. Statin-induced myopathy (SIM) is typically self-limited showing remission in the following weeks or months after statin cessation. Although EMG studies support the presence of typical myopathy features in SIM, it cannot point-out specific changes attributed to a statin-related dysfunction. Patient outcome was favorable on hospital discharge. On a two week check-up, she reported improvement in muscle strength, range of motion and remitted myalgia. Repeated blood work showed a descending trend in both CK and AST, with values in normal range.

Conclusions. The clinical case, the whole algorithm of clinical evaluation and paraclinical tests that lead to final diagnosis and the literature review, highlight the importance of an exhaustive approach. Electrophysiology tests offer important aid to the physician in the approach of patients with an underlying toxic myopathy in initial diagnosis, follow-up and biopsy yield if necessary.
\end{abstract}

Keywords: Inflammatory myopathies, statin induced myotoxicity, electromyography, myositis, 3-hydroxy-3-methylglutaryl-coenzyme A reductase antibodies

\section{INTRODUCTION}

Inflammatory myositis group include polymyositis, dermatomyositis, inclusion body myositis and necrotising myopathy, which typically present with weakness of the arms and legs, pain in the muscles and increased level of serum creatine kinase. The diagnosis of the myositis, requires, besides clinical signs and paraclinical tests assessment, a comprehensive overview of other causes of myopathy.

The potential for myotoxicity has been reported in various drugs. This is to be taken into account since myopathy-associated morbidity can have a significant clinical impact. It is relatively difficult to establish a real incidence of drug-induced myopathy since symptoms may vary significantly and the majority of milder cases are tolerable and remain unreported. Main complains are fatigue, muscle weakness and myalgia accompanied by elevated creatine kinase (CK) serum levels. The more severe forms develop rhabdomyolysis and myoglobinuria (1). Cholesterol-lowering drugs (statins), colchicine, corticosteroids, hydroxychloroquine, amiodarone 
and zidovudine are among the most cited drugs involved in myotoxicity (2). Although symptoms are typically reversible, especially in early stages, chronic toxicity in undiagnosed cases may cause irreversible damage. Being often a diagnosis of exclusion, the role of muscle biopsy remains undisputed as a central tool in differentiating between various muscle tissue lesions. Apart from histopathology exam, electrophysiology study can aid the clinician through a non-invasive screening for a potential drug-induced myopathy at onset and also in followup visits (test for reversibility). We report a case of a 70 year-old patient, treated with statins, presenting muscle weakness, in which electromyography (EMG) features suggested a potential drug-induced myopathy after being correlated with later clinical improvement on no-statin regime.

\section{CASE HISTORY}

We report the case of a 70-year old female patient, admitted to the Rheumatology department for mild to moderate muscle weakness on both shoulder and pelvic girdle with accompanying discrete myalgia. Onset of symptoms occurred in the last year with tolerable myalgia, accompanied by mild muscle weakness shortly after, with progressive worsening in the last couple of months. On physical examination, muscle strength tests showed a grade 4 weakness in proximal muscle groups for both upper and lower extremities. Initially, no drug linked with myotoxicity could be identified in her present out-patient therapy regime, which included a thiazide-like diuretic drug for hypertension, vitamin D supplement and an occasional topic non-steroidal anti-inflammatory agent. She did not have similar symptoms in the past and no relevant personal or hereditary disease history. First lab-work included serum levels for muscle enzymes and autoantibody screening. Results yielded a negative autoimmunity profile and elevated serum levels for $\mathrm{CK}$ of $\mathrm{x} 1.5$ fold normal range, mildly increased aspartate aminotransferase (AST), normal alanine aminotransferase (ALT) and lactate dehydrogenase (LDH). A viral hepatitis was ruled out.
Upon further questioning, additional information was obtained from the out-patient care physician. It was determined that a statin therapy was in fact administered for the past three years and that, on a regular check-up one month prior, interruption of statins was recommended on the basis of muscle symptoms and even more elevated muscle enzymes: CK $x 3$ fold AST $x 2$ fold normal range. Judging by the current results, a favourable trend in myopathy serum markers was noted after cessation of statin therapy. Muscle biopsy was hindered by difficulties in obtaining a patient consent. Autoimmunity panel was the extended to include 3-hydroxy-3-methylglutaryl-coenzyme A reductase (HMGCR) antibodies, which yielded a negative result. We opted for an EMG in order to determine if the potential statin myotoxicity is also expressed through abnormal electrophysiology features.

\section{NEUROPHYSIOLOGIC FINDINGS}

Nerve conduction found no motor or sensory problem in both upper extremity (median motor and radial sensitive) and borderline tibial compound motor action potential with normal sural sensitive response. Corresponding $\mathrm{F}$ responses were normal for the investigated nerves. The needle EMG was abnormal, with diffuse fibrillation potentials in almost all investigated sites, both in the proximal and distal muscles (Table 1). Complex repetitive discharges were also observed in most muscles tested. Most of the motor unit action potentials (MUAPs), had a short duration and high amplitude, indicating an underling neuropathic cause. Some polyphasic and high amplitude MUPs were also seen, furthering the suspicion of a neurogenic cause, however most MUPs were of low amplitude. Recruitment was early and slightly reduced. This mixed pattern was more pronounced in the lower extremity.

Although the neurophysiology findings can suggest a neurogenic underlining cause, the existence of clear myogenic signs on needle EMG revealed the probable cause for the clinical presentation as being myogenic in nature.

TABLE 1. Neurophysiologic findings

\begin{tabular}{|l|l|l|l|l|l|l|l|l|l|l|l|l|}
\hline \multicolumn{1}{|c|}{ Muscle } & Side & Fibs. & $\begin{array}{c}\text { Pos. } \\
\text { Wave }\end{array}$ & Fasc & $\begin{array}{c}\text { MYo. } \\
\text { Disch. }\end{array}$ & $\begin{array}{c}\text { Normal } \\
\text { MUP }\end{array}$ & Poly & $\begin{array}{c}\text { Low } \\
\text { Amp. }\end{array}$ & $\begin{array}{c}\text { High } \\
\text { Amp. }\end{array}$ & Dur. & Recruit & $\begin{array}{c}\text { Int. } \\
\text { Patt. }\end{array}$ \\
\hline Tibialis Ant & $\mathrm{R}$ & +3 & +1 & 0 & 0 & +2 & ++ & +2 & +1 & Short & Early & Reduce \\
\hline Biceps Brahialis & $\mathrm{R}$ & +1 & 0 & 0 & 0 & +3 & + & +1 & 0 & Short & Early & Reduce \\
\hline L5 Paraspinal & $\mathrm{R}$ & +2 & +1 & 0 & 0 & +4 & 0 & 0 & 0 & Short & Reduce & Normal \\
\hline Deltoid & $\mathrm{R}$ & +2 & +1 & 0 & 0 & +3 & + & +1 & 0 & Short & Early & Reduce \\
\hline
\end{tabular}


Patient outcome was favourable on hospital discharge. On a two week check-up, she reported improvement in muscle strength, range of motion and remitted myalgia. Repeated blood work showed a descending trend in both CK and AST, with values in normal range.

\section{DISCUSSION}

Statin-induced myopathy (SIM) is a general term which refers to all muscle-related adverse events that can be attributed to cholesterol lowering therapy. This broader terminology encompasses multiple clinical conditions, ranging from asymptomatic raised $\mathrm{CK}$ and myalgia to myositis and rhabdomyolysis (3). Some authors also advocate that the term "myositis" is not limited to muscle symptoms with raised $\mathrm{CK}$ and should include biopsy-proven muscle inflammation. The PRIMO study, an observational study on 7924 hyperlipidaemia patients, offered insight into the clinical features of SIM. Symptoms included fatigue, myalgia, weakness, muscle tenderness and cramping. It has a similar proximal and symmetrical pattern as in autoimmune myopathies. Median time between medication initiation and onset of muscle symptoms was one month (4). Incidence varies between 5-20\% in observational studies and tends to be lower in randomised trials (5). SIM is typically self-limited showing remission in the following weeks or months after statin cessation. Biopsy studies performed in severe forms of SIM report the presence of a necrotizing autoimmune myopathy, distinct form the typical inflammatory infiltrate seen in polymyositis or dermatomyositis (6).

There are multiple patient-related risk factors for SIM, which include genetic predisposition, female gender, advanced age, low body mass index, impaired renal and hepatic function, hypothyroidism, vitamin E and D deficiency (5). Some studies support the hypothesis of a statin-related risk factor. This is based on fact that lipophilic statins, such as atorvastatin and simvastatin have a higher capacity to penetrate non-hepatic cells, as opposed to hydrophilic statins, such as pravastatin and rosuvastatin. Also, plasma half-life and dose contribute to the potential statin myotoxicity (7), (8).

Pathomechanism of SIM is not fully understood. Several hypothesis that explain the relation between statin use and myopathy include: inhibition of coenzyme Q10 synthesis (impaired respiratory chain of the mitochondria), depletion of sarcolemma mem- brane cholesterol, disturbed calcium metabolism and autoimmunity (5). The majority of patients with asymptomatic or self-limiting SIM do not develop HMGCR antibodies. Upregulation of these antibodies is more frequently seen in necrotizing autoimmune myopathy. Our patient, having also a milder clinical expression of the disease, did not develop autoimmune status, supporting the fact that antibody secretion can be potential prognostic marker.

The diagnostic work-up of any patient with suspicion of an underlying myopathy should include a needle EMG examination. Data obtained through this test is used both as a diagnostic aid and as a mean to better prepare the patient for a more indepth histopathology assessment. A common practice when performing an EMG is to examine one proximal and one distal muscle from both upper and lower extremity and also a paraspinalis muscle. Particularities of the clinical picture may demand additional muscle sites to be assessed. The examiner will always take into account other pathologic processes, such as entrapment neuropathy or a radiculopathy. These chronic neurogenic diseases may confound the EMG results (9). Muscles commonly biopsied are the biceps, deltoid, vasti and tibialis anterior. In general, the weakest muscles will show the most important abnormalities. Thus, a clinical muscle strength examination should be carried out to better guide the EMG test.

A key element of the electrodiagnostic test is the capacity to differentiate between an underlying neuropathic and myopathy process. This is done through the examination of 4 components: insertional activity, spontaneous activity, MUAP morphology and recruitment (10). An increased insertional activity, fibrillation potentials, positive sharp waves (PSWs) and abnormal spontaneous activity may suggest existing muscle membrane irritability. PSW and fibrillation potentials are caused by abnormal generation of action potentials due to loss of innervation by either structural or metabolic disorders. Myopathies associated with membrane instability include inflammatory, toxic and necrotic processes, muscular dystrophies and certain metabolic disorders (9). MUAP classically seen in myopathies are polyphasic, have a short duration, low amplitude and early recruitment, due to the fact that there is a dysfunction of individual muscle fibres with preserved number of available motor units.

Electrodiagnostic features related to spontaneous activity are seen in $75 \%$ patients with inflammatory 
myopathy, while the characteristic MUAP changes are observed in up to $90 \%$ of myositis patients (11). Toxic myopathies, such as those induced by lipidlowering agents, typically present an asymptomatic CK elevation, in which case EMG will show a normal pattern. In rare cases, statin-induced myopathy will develop clinical weakness. EMG studies of these patients will yield abnormal patterns in the affected muscle groups, in the form of PSWs, fibrillations and early recruitment of short MUAPs (12).

In one study, Mohammadi A et al, described significant differences of MUAPs parameters and number of phases between statin users and control group (13). Although EMG studies support the presence of typical myopathy features in SIM, it cannot pointout specific changes attributed to a statin-related dysfunction. Nevertheless, use of EMG in SIM is should be a common practice in addressing patients

\section{$\overline{\text { REFERENCES }}$}

1. Valiyil R., Christopher-Stine L. Drug-related Myopathies of Which the Clinician Should Be Aware. Current rheumatology reports. 2010; 12(3):213-220. doi:10.1007/s11926-010-0104-3.

2. Dalakas M.C. Toxic and drug-induced myopathies. J Neurol Neurosurg Psychiatry. 2009; 80(8):832-8. doi:10.1136/ jnnp.2008.168294.

3. Sathasivam S., Lecky B. Statin induced myopathy. BMJ. 2008; 337:a2286.

4. Bruckert E., Hayem G., Dejager S. et al. Mild to moderate muscular symptoms with high-dosage statin therapy in hyperlipidemic patients - the PRIMO study. Cardiovasc Drugs Ther 2005; 19:403-14.

5. Sathasivam S. Statin induced myotoxicity. Eur J Intern Med. 2012; 23:317-324. doi: 10.1016/j.ejim.2012.01.004.

6. Grable-Esposito P., Katzberg H.D., Greenberg S.A., Srinivasan J., Katz J., Amato A.A. Immune-mediated necrotizing myopathy associated with statins. Muscle Nerve. 2010; 41(2):185-190.

7. Silva M., Matthews M.L., Jarvis C. et al. Meta-analysis of druginduced adverse events associated with intensive-dose statin therapy. Clin Ther 2007; 29:253-60. with a potential SIM. Because the vast majority of cases will have a self-limited disease course, CK and EMG can aid the physician in monitoring the disease progression and statin withdrawal effect. Expert groups do not recommend routinely muscle biopsy for assessment of muscle damage in SIM (14). Biopsy should be limited to persistent forms in which a differential diagnosis in mandatory.

\section{CONCLUSION}

Differential diagnosis in myopathies requires extensive studies, between which, electrophysiology tests offer important aid to the physician. EMG can correlate the present symptoms with an underlying myopathic process and it can be used as an additional follow-up exam, to evaluate treatment response. In the same time, can optimise the muscle biopsy yield when necessary.

Conflict of interest: none declared Financial support: none declared

8. Kuncl R.W. Agents and mechanisms of toxic myopathy. Curr Opin Neurol. 2009; 22(5):506-515.

9. Paganoni S., Amato A. Electrodiagnostic evaluation of myopathies. Phys Med Rehabil Clin N Am. 2013; 24:193-207.

10. Lacomis D. Electrodiagnostic approach to the patient with suspected myopathy. Neurol Clin. 2002; 20(2):587-603.

11. Gilchrist J.M., Sachs G.M. Electrodiagnostic studies in the management and prognosis of neuromuscular disorders. Muscle Nerve. 2004; 29:165-90.

12. Meriggioli M.N., Barboi A.C., Rowin J., Cochran E.J. HMG-CoA Reductase Inhibitor Myopathy: Clinical, Electrophysiological, and Pathologic Data in Five Patients. J. Clin. Neuromuscul. Dis. 2001; 2:129-134.

13. Abdulrazaq M., Hamdan F., Al Tameemi W. Electrophysiologic and clinico-pathologic characteristics of statin-induced muscle injury. Iran J Basic Med Sci 2015;18:737.

14. Thompson P.D., Clarkson P.M., Rosenson R.S., National Lipid Association Statin Safety Task Force Muscle Safety Expert Panel An assessment of statin safety by muscle experts. Am J Cardiol. 2006; 97:69C-76C. 